\title{
Effects of Pre-Germinated Brown Rice on $\beta$-Amyloid Protein-Induced Learning and Memory Deficits in Mice
}

\author{
Takayoshi Mamiya, ${ }^{a}$ Takamasa Asanuma, ${ }^{a}$ Mitsuo Kise, ${ }^{b}$ Yukihiko Ito, ${ }^{b}$ Aya Mizukuchi, ${ }^{b}$ \\ Hiromichi Aото, ${ }^{b}$ and Makoto UKaI ${ }^{*, a}$ \\ ${ }^{a}$ Department of Chemical Pharmacology, Faculty of Pharmacy, Meijo University; 150 Yagotoyama, Tempaku-ku, Nagoya \\ 468-8503, Japan: and ${ }^{b}$ Cereal Utilization Laboratory, Applied Food Research Division, Central Research Center, FANCL \\ Co., Ltd.; 12-13 Kamishinano, Totsuka-ku, Yokohama 244-0806, Japan. \\ Received January 21, 2004; accepted April 6, 2004; published online April 8, 2004
}

We evaluated the effects of pre-germinated brown rice (hatsuga genmai, PGR) on learning and memory and compared them with those of polished rice or cornstarch. In mice that were fed pellets of polished rice or PGR for two weeks, the learning ability in the Morris water maze test was significantly enhanced compared with mice that were fed cornstarch pellets. In the Y-maze test, the intake of food pellets for two weeks failed to affect spontaneous alternation behavior. $\beta$-Amyloid ${ }_{25-35}\left(\mathrm{~A} \beta_{25-35}: 3 \mathrm{nmol} / \mathrm{mouse}\right.$, i.c.v. $)$ protein impaired spontaneous alternation behavior in mice that were fed pellets of cornstarch or polished rice. In contrast, PGR pellets prevented the $A \beta_{25-35}$-induced impairment of spontaneous alternation behavior. These results suggest that polished rice and PGR have facilitating effects on spatial learning. In particular, it is surmised that PGR may prevent Alzheimer's disease associated with $\mathbf{A} \beta$.

Key words Alzheimer's disease; learning and memory; pre-germinated brown rice; preventive effect

In Asian countries, polished rice is one of the main staples and is believed to contribute to good health. Rice consists of endosperm, bran layer and germ. Most Japanese like polished rice and eat it every day. Polished rice, however, has no bran layer or a germ that is abundant in nutrients including dietary fiber, vitamins and minerals. On the other hand, pregerminated brown rice (PGR) has become popular in Japan. PGR is brown rice soaked in water to induce slight germination and is known to contain abundant nutrients, namely, approximately 13 times the amount of oryzanol and 15 times that of $\gamma$-aminobutyric acid (GABA) in polished rice. However, it remains to be clarified if those nutrients improve brain function, particularly learning and memory.

Alzheimer's disease is the most common cause of progressive decline in cognitive function among the elderly, and is characterized by the presence of numerous senile plaques and neurofibrillary tangles resulting in neuronal loss. The $\beta$ amyloid $(\mathrm{A} \beta)$ peptide that comprises senile plaques induces brain dysfunction, as evidenced by neurodegeneration and learning and memory impairment in rodents. ${ }^{1-6)}$ Several reports have demonstrated that $\mathrm{A} \beta_{1-40}, \mathrm{~A} \beta_{1-42}$ and $\mathrm{A} \beta_{25-35}$ proteins impaired learning and memory to the same extent. ${ }^{1,45)}$ Similarly, Terranova et al. have shown that those three proteins induce the cognitive dysfunction in rats. $\left.{ }^{6}\right)$ These changes are reminiscent of the deposition of amyloid plaques endogenously constituted from $\mathrm{A} \beta_{1-40}$ and $\mathrm{A} \beta_{1-42}$ proteins, but not $\mathrm{A} \beta_{25-35}$, the extent of which is correlated with progressive cognitive deficit and memory impairment as observed in Alzheimer's disease patients. ${ }^{7)}$

Therefore, we evaluated the effects of PGR on learning and memory and compared them with those of polished rice or cornstarch (as standard), by feeding mice pellets containing each extract. The protective effects of PGR on $\mathrm{A} \beta_{25-35^{-}}$ induced impairment of learning and memory were assessed in three different tasks.

\section{MATERIALS AND METHODS}

Animals and Foods Five-week-old male ICR mice (Nihon SLC Co., Ltd., Shizuoka, Japan) were purchased and food pellets were adequately given to them. The animals were housed in a controlled environment $\left(23 \pm 1^{\circ} \mathrm{C}, 50 \pm 5 \%\right.$ humidity) and were given access to water ad libitum. The room lights were on between $7 \mathrm{~h} 30$ and $19 \mathrm{~h} 30$. We used standard food pellet (AIN-93G, Oriental Yeast Co. Ltd., Japan) containing cornstarch. Each of the food pellets contained standard nutrients and only cornstarch was replaced with either polished rice or PGR (Table 1). The experimental schedule is shown in Fig. 1. All experiments were performed in accordance with the Guidelines for Animal Experiments of Meijo University and the Guiding Principles for the Care and Use of Laboratory Animals approved by the Japanese Pharmacological Society (1987).

$\mathbf{A} \boldsymbol{\beta}_{25-35}$ Treatment $\mathrm{A} \boldsymbol{\beta}_{25-35}$ (Bachem AG, Budendorf, Switzerland) was dissolved in sterile distilled water at a concentration of $1 \mathrm{mg} / \mathrm{ml}$ and allowed to aggregate by incubation at $37^{\circ} \mathrm{C}$ for $4 \mathrm{~d}$ as reported previously. ${ }^{3)}$ The peptide and the

Table 1. Standard Ingredients in Food Pellet

\begin{tabular}{lc}
\hline \multicolumn{1}{c}{ AIN-93G } & Ingredient (\%) \\
\hline Cornstarch & 39.7 \\
Casein & 20 \\
L-Cystine & 0.3 \\
$\alpha$-Cornstarch & 13.2 \\
Sucrose & 10 \\
Soy bean oil & 7 \\
Cellulose powder & 5 \\
Mineral mix & 3.5 \\
Vitamin mix & 1 \\
Choline bicitrates & 0.25 \\
Butylhydroquinone & 0.0014
\end{tabular}

AIN-93G: standard food pellet (Oriental Yeast Co. Ltd., Japan) Cornstarch is replaced by polished rice or PGR. $\alpha$-Cornstarch is added to solidify food pellet. 
vehicle $(3 \mu \mathrm{l})$ were administered gradually intracerebroventricularly (i.c.v.) within approximately $20 \mathrm{~s}$ under light ether anesthesia. Mice exhibited normal behavior within $1 \mathrm{~min}$ after injection. The site of administration was checked by injecting Indian ink in preliminary experiments. Neither insertion of the needle nor injection of the vehicle had a significant influence on survival, behavioral response or cognitive function.

Water Maze Test ${ }^{8)}$ The pool was $1.2 \mathrm{~m}$ in diameter and was made of polypropylene polished plastic, and water in the pool was kept at $18.5 \pm 0.5^{\circ} \mathrm{C}$. The test mice were kept in shelves underneath the pool to eliminate directional olfactory and auditory cues. The plexiglas platform was $10 \mathrm{~cm}$ in diameter and its surface was $2 \mathrm{~cm}$ below the water surface to help the mice climb onto it. The room had adjustable indirect illumination and a camera was fixed to the ceiling. Swimming paths were tracked by the camera and stored in a computer (Target/2 system, Neuroscience Inc., Tokyo, Japan). The mice were not allowed to swim in the pool before training (i.e., there was no habituation). During training in the hidden platform test of the water maze, the platform was not marked by any cues and it was kept in the same place throughout training. In one block of trials, the starting position of the mice was varied pseudorandomly to ensure that three starting positions were used. A mouse that found the platform was allowed to remain there for $30 \mathrm{~s}$ and then returned to its home cage. For mice that were unable to find the platform within $90 \mathrm{~s}$, the training session was terminated and a maximum score of $90 \mathrm{~s}$ was assigned. Training was conducted for seven consecutive days, three times a day. A transfer test was conducted seven days after the $\mathrm{A} \beta_{25-35}$ injection, and the mice swam for $45 \mathrm{~s}$ in the absence of the platform.

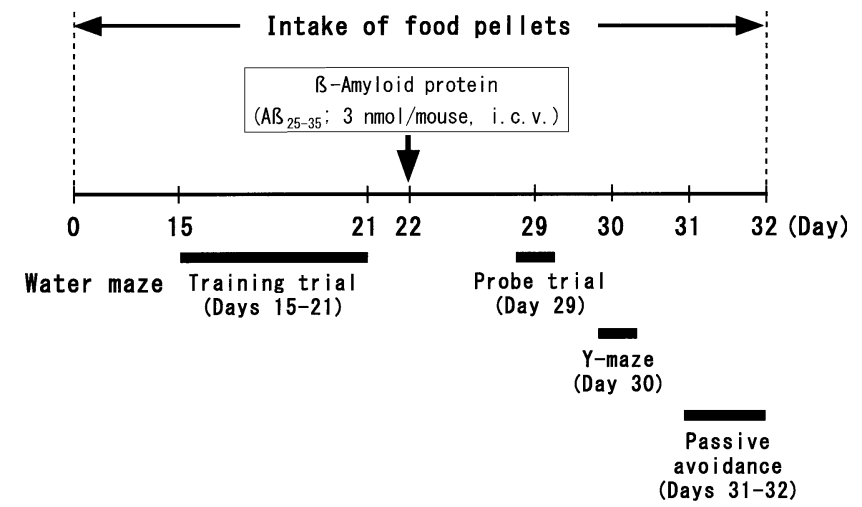

Fig. 1. Experimental Schedule
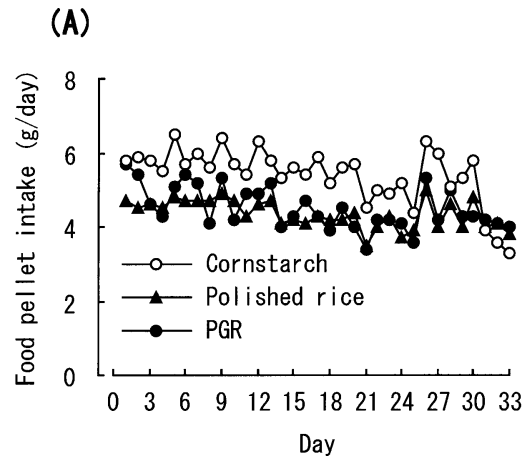

Y-Maze Test $^{9)}$ Short-term memory was examined by monitoring spontaneous alternation behavior in the Y-maze. The maze was made of wood painted black and each arm was $40 \mathrm{~cm}$ long, $12 \mathrm{~cm}$ high, $3 \mathrm{~cm}$ wide at the bottom and $10 \mathrm{~cm}$ wide at the top. The arms converged at an equilateral triangular central area that was $4 \mathrm{~cm}$ at its longest axis. The apparatus was placed on the floor of the experimental room and was illuminated with a $100 \mathrm{~W}$ bulb from $200 \mathrm{~cm}$ above. Each mouse was placed at the end of one arm and allowed to move freely through the maze during an 8-min session, and the series of arm entries was recorded visually. The alternation behavior was defined by the successive entry into the three arms, on overlapping triplet sets, and such behavior (\%) was expressed as the ratio of actual alternations to possible alternations (defined as the total number of arm entries minus two), multiplied by 100 . After the experiments, we counted the number of arm entries, urinations and feces.

Step-through Type Passive Avoidance Test ${ }^{8)}$ This test consisted of two trials. In the acquisition trial, the mice were individually placed in lighted compartments. Ten seconds later, the door to a dark compartment was opened, and the latency until the mouse entered the dark compartment was recorded. After the mouse had stepped through the door, the door was closed and an electric shock $(0.2 \mathrm{~mA}, 2 \mathrm{~s}$, Shockgenerator-Scrambler, Neuroscience Idea Co. Ltd., Osaka, Japan) was delivered via the grid floor. The mouse was removed after receiving the footshock and returned to the light compartment by the experimenter. The door was re-opened $10 \mathrm{~s}$ later to start the next trial. Training continued in this manner until the mouse stayed in the light compartment for more than $120 \mathrm{~s}$ in a single trial. Twenty-four hours later, each animal was placed in the light compartment and the step-through latency was recorded until $300 \mathrm{~s}$ had elapsed (retention trial).

Statistical Analysis All results were expressed as means \pm S.E.M. for each group. Statistical analysis of difference among the three groups was conducted with Dunnett's multiple comparison test and that between two groups, with the Student's $t$-test. The level of significant difference was taken at $p<0.05$.

\section{RESULTS}

Food Intake and Body Weight Figure 2 shows the average food pellet intake and changes in body weight during the experimental period. Although not significant, more cornstarch pellets were ingested than polished rice or PGR pellets

(B)

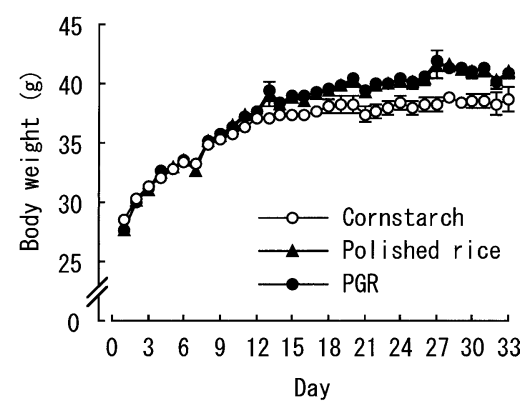

Fig. 2. Changes in Food Pellet Intake (A) and Body Weight (B) During the Experimental Period 

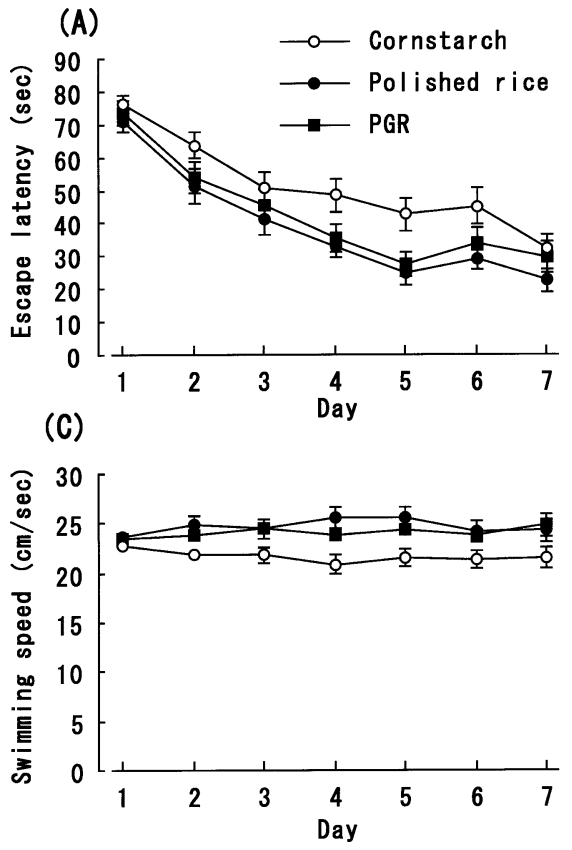

(B)

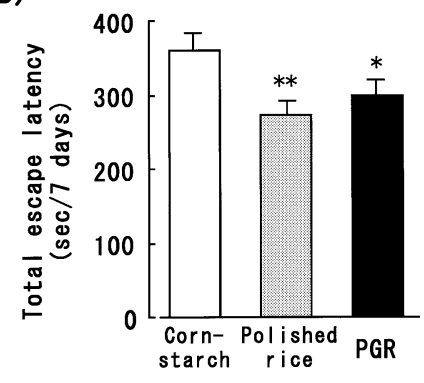

Fig. 3. Performance in Morris Water Maze Test of Mice Fed Food Pellets

PGR: pre-germinated brown rice. A and B: Escape latency and total escape latency in the hidden platform test. $* p<0.05 ; * * p<0.01 v s$. cornstarch group (Dunnett's multiple comparison test). C: Swimming speed in the hidden platform test. The moving distances for $90 \mathrm{~s}$ were calculated from the recorded swimming paths.

(A)

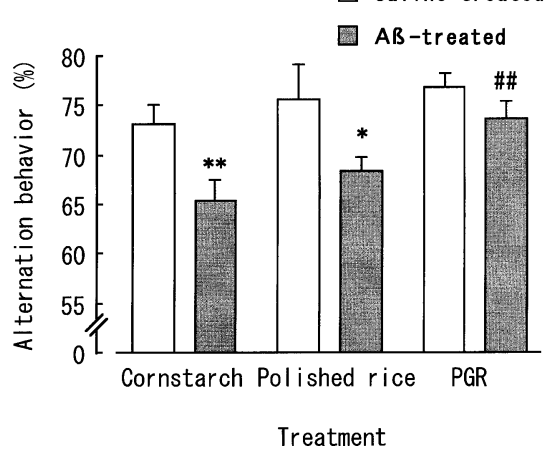

(B)

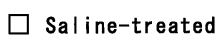

圆 $A ß-$ treated

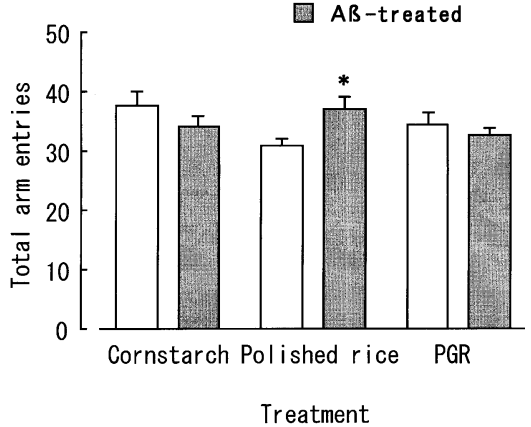

Fig. 4. Effects of Food Pellets on $\mathrm{A} \beta_{25-35}$-Induced Impairment of Spontaneous Alternation Behavior (A) and Total Arm Entries (B) in Mice ( $n=17$ or 18$)$ PGR: pre-germinated brown rice. $* p<0.05, * * p<0.01 v s$. corresponding saline-treated mice (Student's $t$-test), \#\# $p<0.01 v s$. cornstarch $+\mathrm{A} \beta$-treated mice (Dunnett's multiple comparison test).

(Fig. 2A). On the other hand, both polished rice and PGR groups gained more weight than the cornstarch group (Fig. 2B).

Water Maze Test Figure 3 shows the performance in the Morris water maze test. The escape latencies of the cornstarch group were slowly shortened by repeated training, whereas those of the polished rice and PGR groups were rapidly shortened (Fig. 3A). The total escape latencies of both polished rice and PGR groups were reduced significantly compared with that of the cornstarch group (Fig. 3B). In addition, mice that were fed polished rice and PGR pellets swam faster than those that were fed cornstarch pellets in all training days except day 1 (Fig. 3C). Seven days after training, the mice were subjected to a 45-s transfer test without the goal platform. In the present study, however, even the A $\beta_{25-35}$ peptide did not induce any impairment in the transfer test (data not shown).

Y-Maze Test Eight days after the $\mathrm{A} \beta_{25-35}$ peptide injec- tion, the Y-maze test was carried out (Figs. 4A, B). There was no significant difference in the spontaneous alternation behavior or the total arm entries among the three salinetreated groups. In the cornstarch and polished rice groups, the $\mathrm{A} \beta_{25-35}$ peptide impaired spontaneous alternation behavior. In contrast, PGR pellets prevented the impairment of spontaneous alternation behavior without affecting total arm entries. The numbers of urinations and feces were similar among the three mouse groups.

Step-through Type Passive Avoidance Test Table 2 shows the results of performance measurement in the acquisition trial on day 1 and the retention trial on day 2 of the step-through type passive avoidance test. Despite the A $\beta_{25-35}$ peptide injection, we could not observe any significant changes in the acquisition or the retention trial, and there was little difference in both trials among the three mouse groups. 
Table 2. Performance of Mice in Multi-trial Passive Avoidance Test

\begin{tabular}{|c|c|c|c|c|}
\hline \multirow{2}{*}{ Treatment } & \multirow{2}{*}{$\mathrm{N}$} & \multicolumn{2}{|c|}{ Acquisition trial } & \multirow{2}{*}{$\begin{array}{c}\text { Retention trial } \\
\text { Step-through latency }(\mathrm{s})^{c)}\end{array}$} \\
\hline & & No. of trials ${ }^{a)}$ & Latency (s) on the first trial ${ }^{b)}$ & \\
\hline \multicolumn{5}{|l|}{ Cornstarch } \\
\hline Saline & 15 & $3.5 \pm 0.3$ & $9.6 \pm 2.2$ & $266.1 \pm 12.7$ \\
\hline $\mathrm{A} \beta_{25-35}$ & 16 & $4.4 \pm 0.7$ & $6.5 \pm 0.9$ & $235.1 \pm 21.9$ \\
\hline \multicolumn{5}{|l|}{ Polished rice } \\
\hline Saline & 8 & $3.1 \pm 0.4$ & $9.0 \pm 1.4$ & $253.6 \pm 24.4$ \\
\hline $\mathrm{A} \beta_{25-35}$ & 16 & $4.7 \pm 0.6$ & $6.5 \pm 0.9$ & $241.7 \pm 18.9$ \\
\hline \multicolumn{5}{|l|}{ PGR } \\
\hline Saline & 8 & $3.0 \pm 0.2$ & $10.3 \pm 3.3$ & $223.3 \pm 30.0$ \\
\hline $\mathrm{A} \beta_{25-35}$ & 17 & $3.8 \pm 0.4$ & $7.4 \pm 1.8$ & $249.2 \pm 23.3$ \\
\hline
\end{tabular}

a) Number of trials until the mouse stayed in the light compartment for more than $120 \mathrm{~s}$. b) Latency until the mouse entered the dark compartment from the light compartment in the first training. N indicates the number of mice used. c) Step-through latency on day 2 (cut-off time: $300 \mathrm{~s}$ ).

\section{DISCUSSION}

In this study, we evaluated the effects of PGR on learning and memory and compared them with those of polished rice or cornstarch. To our knowledge, this is the first report of the behavioral effects of PGR on learning and memory.

Polished rice and PGR were found to enhance learning ability in the water maze test. This test is well used and is considered to be an important index of spatial learning. The swimming speed of the cornstarch group is lower than those of the polished rice and PGR groups. We also compared the swimming paths among the three groups. The results are similar to the case of escape latency, while, in other words, both rice groups swam shorter than cornstarch group on days 4-6. There was no difference in escape latency, swimming speed and swimming paths on the day 1 among three groups, suggesting that the memorization in both groups of polished rice and PGR is faster than that of cornstarch group, and that mice fed polished rice and PGR are eager to reach the goal platform. Additionally, the swimming speed of cornstarch group is similar to that of control mice in our previous report. ${ }^{8)}$ Taken together, polished rice and PGR enhanced the learning ability, resulting in faster swimming. During this experiment, the food pellet intake of the cornstarch group was higher than that of the other two groups; however, the increase in body weight of that group was smaller. As shown in Table 1, cornstarch was the only ingredient that was replaced in the PGR and polished rice pellets; thus, it is possible that the results were dependent on the nutritive value of PGR or polished rice. In fact, cornstarch we used is purified and consists of amylopectin and amylose, which are carbohydrates, indicating that this is a merely source of energies. On the other hand, rice contains vitamins, minerals and amino acids besides starch. Thus, polished rice and PGR heightened the ability of learning and memory whereas we cannot specify the nutritive factor here. We also checked motor coordination in the rotarod test, but found no significant difference among the three groups (data not shown), indicating that polished rice and PGR facilitate spatial learning without any motor deficits.

Concerning the Y-maze test, which is considered to reflect short-term and working memory in human, the three groups treated with saline showed similar spontaneous alternation behavior. On the other hand, although the spontaneous alternation behavior of both cornstarch and polished rice groups was impaired by $\mathrm{A} \beta_{25-35}$, no such impairment was observed in the PGR group without changing total arm entries. Moreover, there were no significant differences regarding the numbers of urinations and feces, demonstrating that those food pellets failed to affect emotional responses.

We could not determine the effects of polished rice and PGR in the passive avoidance test because this test had a cutoff time $(300 \mathrm{~s})$ so that the ceiling effect occurred. Moreover, even $A \beta_{25-35}$ treatment did not shorten the step-through latency, and thus we were unable to evaluate the effects of the food pellets in this test. Altogether, the results suggest that PGR may be a very useful food for activating brain function.

Several mechanisms may contribute to the apparent positive effects of polished rice and PGR on learning and memory. PGR contains a large amount of GABA, which plays a crucial role in memory processes. One recent work has shown that GABA receptors regulate calcineurin-mediated long-term depression (calcineurin is one of the neuronal plasticity molecules associated with learning and memory), and this phenomenon underlies the increased excitability of hippocampal CA1 neurons associated with long-term potentiation. ${ }^{10)}$ It is reported that GABA is carried into the brain through the blood brain barrier. ${ }^{11)}$ It is considered that the transportation to the brain is important rather than the actual volume that GABA permeates. Very recently, it has been reported that GABA and its receptor agonist improve cognitive decline that accompanies aging in monkeys. ${ }^{12)}$ Because the glutamatergic system is one of most important neuroregulatory systems in learning and memory, we attempted to detect it in the brain of mice fed PGR, and found that CaMKII $\alpha$ phosphorylation, which is activated by mediation with NMDA receptors, was enhanced compared with the other groups (unpublished observation). Taken together, the large amount of GABA in PGR may regulate the glutamatergic system by enhancing glutamate release and/or the sensitivity of NMDA receptors, resulting in memory enhancement.

PGR contains approximately 13 times the amount of oryzanol in polished rice. Ferulic acid ester is one of main components of oryzanol, a phytosterol derived from rice bran. Recently, it has been reported that the long-term administration ferulic acid acts to protect against $\mathrm{A} \beta_{1-42}$-induced neurotoxicity and memory disturbance ${ }^{13)}$ as well as learning and memory impairment induced by scopolamine or cycloheximide. ${ }^{14,15)}$ Previous reports have hypothesized that oxidative stress is one of the mechanisms of $\mathrm{A} \beta$-induced 
neurotoxicity. In vitro studies have shown that $\alpha$-tocopherol, a representative antioxidant, inhibits $\mathrm{A} \beta$-induced neuronal cell death and lipid peroxidation. ${ }^{16-18)}$ Ferulic acid also possesses free radical scavenging activity and reduces peroxidative damage. ${ }^{19,20)}$ Thus, PGR may ameliorate $\mathrm{A} \beta_{25-35}$-induced learning and memory deficits by scavenging oxidative stress.

Several years ago, PGR has been reported to inhibit prolyl endopeptidase (PEP; EC 3.4.21.26) activity in vitro by Dr. H. Kayahara of Shinshu University, Japan (unpublished results). PEP is a serine endopeptidase that is widely distributed in mammalian tissues and specifically cleaves peptide bonds at the carboxylic end of proline residues of oligopeptides. ${ }^{21)}$ This enzyme has been identified as a putative $A \beta$-generating enzyme and its activity is increased in Alzheimer's disease patients. ${ }^{7)}$ These findings indicate that the inhibitory effect of PGR on PEP may be a direct evidence of memory enhancement in the mice observed in this study. Although we raised some possible mechanisms above, maybe the combination of those possibilities but not only one is most important in the enhancement of learning ability or prevention of the memory impairment.

It is concluded that polished rice or PGR has facilitating effects on spatial learning. In particular, it is surmised that therapeutically PGR may prevent Alzheimer's disease associated with $\mathrm{A} \beta$ whereas it is unclear whether neuronal damages are recovered. However, the experimental period may be too short to examine in detail the effects of rice and therefore, further detailed studies are needed. Moreover, a comparison of brown rice with PGR as our next step will confirm present data.

Acknowledgements This study was supported in part by Grants-in Aid for Scientific Research, High-Tech Research Center Project and Scientific Frontier Research Project from Ministry of Education, Culture, Sports, Science and Technology, Japan, and the Sasakawa Scientific Research Grant from
The Japan Science Society.

\section{REFERENCES}

1) Nitta A., Itoh A., Hasegawa T., Nabeshima T., Neurosci. Lett., 170, 63-66 (1994).

2) Nitta A., Fukuta T., Hasegawa T., Nabeshima T., Jpn. J. Pharmacol., 73, 51-77 (1997).

3) Maurice T., Su T. P., Privat A., Neuroscience, 83, 413-428 (1998).

4) Yamada K., Tanaka T., Mamiya T., Shiotani T., Kameyama T., Nabeshima T., Br. J. Pharmacol., 126, 235-244 (1999).

5) Olariu A., Tran M. H., Yamada K., Mizuno M., Hefco V., Nabeshima T., J. Neural. Transm., 108, 1065-1079 (2001).

6) Terranova J. P., Kan J. P., Storme J. J., Perreaut P., Le Fur G., Soubrie P., Neurosci. Lett., 213, 79-82 (1996).

7) Selkoe D. J., Neuron, 6, 487-498 (1991).

8) Manabe T., Noda Y., Mamiya T., Katagiri H., Houtani T., Nishi M., Noda T., Takahashi T., Sugimoto T., Nabeshima T., Takeshima H., Nature (London), 394, 577-581 (1998).

9) Ukai M., Katoh T., Mamiya T., Neuroreport, 12, 3723 -3727 (2001).

10) Lu Y. M., Mansuy I. M., Kandel E. R., Roder J., Neuron, 26, 197205 (2000).

11) Garcia S. C., Moretti M. B., Garay M. V., Batlle A., Gen. Pharmacol., 31, 579-582 (1998).

12) Leventhal A. G., Wang Y., Pu M., Zhou Y., Ma Y., Science, 300, $812-815$ (2003).

13) Yan J. J., Cho J. Y., Kim H. S., Kim K. L., Jung J. S., Huh S. O., Suh H. W., Kim Y. H., Song D. K., Br. J. Pharmacol., 133, 89-96 (2001).

14) Hiraga Y., Nakata N., Jin H., Ito S., Sato R., Yoshida A., Mori T., Ozeki M., Ikeda Y., Arzneimittelforschung, 43, 715-721 (1993).

15) Hsieh M. T., Tsai F. H., Lin Y. C., Wang W. H., Wu C. R., Planta Med., 68, 754-756 (2002).

16) Behl C., Davis J. B., Lesley R., Schubert D., Cell, 77, 817-827 (1994).

17) Schubert D., Behl C., Lesley R., Brack A., Dargusch R., Sagara Y., Kimura H., Proc. Natl. Acad. Sci. U.S.A., 92, 1989-1993 (1995).

18) Mark R. J., Pang Z., Geddes J. W., Uchida K., Mattson M. P., J. Neurosci., 17, 1046-1054 (1997).

19) Scott B. C., Aruoma O. I., Evans P. J., O’Neill C., Van der Vliet A., Cross C. E., Tritschler H., Halliwell B., Free Radic. Res., 20, 119133 (1994).

20) Zheng R. L., Zhang H., Free Radic. Biol. Med., 22, 581-586 (1997).

21) Yoshimoto T., Fischl M., Orlowski R. C., Walter R., J. Biol. Chem., 253, 3708-3716 (1978). 